\title{
Adsorption Behavior of Arabinogalactan-proteins
}

\section{(AGPs) from Acacia senegal Gum at Solid-Liquid}

\section{Interface}

Athénaïs Davantès ${ }^{1 *}$, Michaël Nigen ${ }^{2}$, Christian Sanchez ${ }^{2}$, and Denis Renard ${ }^{1}$

${ }^{1}$ INRAE Pays de la Loire, UR BIA, 3 impasse Yvette Cauchois, La Géraudière, CS 71627, F44316 Nantes Cedex 3, France

${ }^{2}$ UMR IATE, UM-INRAE-CIRAD-Montpellier Supagro, 2 Place Viala, F-34060 Montpellier Cedex, France 
Table S1. Biochemical composition and structural parameters of A. senegal gum and its fractions

\begin{tabular}{|c|c|c|c|c|c|c|}
\hline & HIC-F1 & HIC-F2 & HIC-F3 & $\begin{array}{c}\text { IEC-F1 } \\
\text { (FA) }\end{array}$ & $\begin{array}{c}\text { IEC-F2 } \\
\text { (FD) }\end{array}$ & $\begin{array}{c}\text { A. } \\
\text { senegal }\end{array}$ \\
\hline Sugar (\%) & 96.4 & 91.8 & 81.3 & 86.1 & 94.5 & 94.4 \\
\hline Arabinose & 26.8 & 35.6 & 38.3 & 35.5 & 26.1 & 29.8 \\
\hline Galactose & 39.0 & 34.4 & 33.3 & 33.5 & 40.5 & 38.5 \\
\hline Rhamnose & 12.5 & 13.7 & 13.9 & 12.8 & 12.4 & 12.8 \\
\hline Glucuronic acid & 20.3 & 15.6 & 13.7 & 17.3 & 19.8 & 17.9 \\
\hline 4-O-Me-Glucuronic acid & 1.4 & 0.6 & 0.7 & 1.0 & 1.2 & 1.0 \\
\hline Protein $(\%)^{a}$ & 0.5 & 6.3 & 13.8 & 11.5 & 1.6 & $2.2^{b}$ \\
\hline Mineral (\%) & 3.0 & 1.9 & 4.9 & 2.4 & 4.0 & 3.4 \\
\hline Net Charge $\mathbf{N}_{\mathrm{m}}{ }^{\mathrm{c}}$ & 357 & 1156 & 1219 & 2691 & 514 & 572 \\
\hline $\begin{array}{l}\text { Average molecular weight } \\
\left(M_{w}, \text { g.mol }^{-1}\right)\end{array}$ & $3.5 \times 10^{5}$ & $1.5 \times 10^{6}$ & $1.6 \times 10^{6}$ & $3.1 \times 10^{6}$ & $5.3 \times 10^{5}$ & $6.5 \times 10^{5}$ \\
\hline $\begin{array}{ll}\text { Polydispersity } & \text { index } \\
\left(M_{w} / M_{n}\right) & \\
\end{array}$ & 1.4 & 1.3 & 1.9 & 1.2 & 1.9 & 2.2 \\
\hline High $M_{w}$ AGP (\%) & 3 & 72.7 & 55.6 & 97.4 & 11.5 & 15.4 \\
\hline Intrinsic viscosity $\left(\mathrm{mL} \cdot \mathrm{g}^{-1}\right)^{\mathrm{d}}$ & 16.2 & 64.3 & 54.7 & 87.8 & 29.2 & 30.2 \\
\hline $\begin{array}{l}\text { Partial specific volume } v_{s}^{\circ} \\
\left(\mathrm{cm}^{3} \cdot \mathrm{g}^{-1}\right)\end{array}$ & 0.5616 & 0.5876 & 0.6500 & 0.6095 & 0.5823 & 0.5870 \\
\hline Density & 1.781 & 1.702 & 1.538 & 1.641 & 1.717 & 1.703 \\
\hline $\begin{array}{l}\text { Partial specific adiabatic } \\
\text { compressibility coefficient } \\
\beta_{\mathrm{s}}^{\circ}\left(\mathbf{x}^{10} \mathbf{1 0}^{11} \cdot \mathrm{Pa}^{-1}\right)\end{array}$ & -18.3 & -14.4 & -1.0 & -9.4 & -12.9 & -12.2 \\
\hline Hydrophobicity index & -1.46 & -1.01 & -1.14 & -1.39 & -1.15 & -1.18 \\
\hline $\begin{array}{l}R_{g}(n m) \\
(\% \text { of macromolecules } \\
\text { considered for the } \mathbf{R g})\end{array}$ & 6.5 & $\begin{array}{c}30 \\
(95 \%)\end{array}$ & $\begin{array}{c}36 \\
(65 \%)\end{array}$ & $\begin{array}{c}46 \\
(71 \%)\end{array}$ & $\begin{array}{c}23.0 \\
(37 \%)\end{array}$ & $\begin{array}{c}30 \\
(49 \%)\end{array}$ \\
\hline $\begin{array}{l}R_{h}(n m) \\
\text { Dynamic light scattering }\end{array}$ & 9.1 & $17^{\mathrm{e}}, 24^{\mathrm{f}}$ & $17^{\mathrm{e}}, 23^{\mathrm{f}}$ & $30^{\mathrm{e}}, 32^{\mathrm{f}}$ & $13^{\mathrm{e}}, 14^{\mathrm{f}}$ & $15^{\mathrm{e}}, 16^{\mathrm{f}}$ \\
\hline
\end{tabular}

HIC : hydrophobic interaction chromatography ; IEC : ion exchange chromatography

Data adapted from Apolinar-Valiente et al. (2019) Food Hydrocolloids 89 pp. 864-873; Sanchez et al. (2018) Food Hydrocolloids 78 pp 140160; Sanchez et al. (2008) Biophysical Journal 94(2) pp 629-639 and Tamayo et al. (2018) Colloids Interfaces 2 pp 11. a Protein content was measured using the Kjeldhal method; ${ }^{b}$ Percentage of nonpolar aminoacids; ${ }^{c}$ Computed from biochemical compositions (uronic acids and charged aminoacid); ${ }^{\mathrm{d}}$ Measured using differential capillary viscometry (on line size exclusion chromatography system) in $100 \mathrm{mM}$ LiNO ${ }_{3}$ (pH 5.0) solution containing $0.02 \% \mathrm{NaN}_{3} ;{ }^{\mathrm{e}}$ Hydrodynamic radius $\left(\mathrm{R}_{\mathrm{h}}\right)$ in sodium acetate $10 \mathrm{mM} \mathrm{pH} 5$; ${ }^{\mathrm{f}}$ Hydrodynamic radius $\left(\mathrm{R}_{\mathrm{h}}\right)$ in $100 \mathrm{mM}$ $\mathrm{LiNO}_{3}(\mathrm{pH} 5.0)$ 
Table S2. QCM-D and SPR calculated results, protein content (\%),High $\mathrm{M}_{\mathrm{w}}$ AGP (\%), Calculated film density and water content $\left(\Gamma_{\mathrm{H} 2 \mathrm{O}}, \%\right)$ of HIC fractions (F1, F2 and F3), IEC fractions (FA and FD) and A. senegal gum during adsorption on gold surface for a constant $\mathrm{pH} 5.0$ and ionic strength of $10 \mathrm{mM}$.

\begin{tabular}{|c|c|c|c|c|c|c|}
\hline $\begin{array}{l}10 \mathrm{mM} \\
\text { pH } 5.0\end{array}$ & HIC-F1 & HIC-F2 & HIC-F3 & IEC-F1 (FA) & IEC-F2 (FD) & A. senegal \\
\hline Protein content & $0.49 \%$ & $6.3 \%$ & $13.77 \%$ & $11.49 \%$ & $1.59 \%$ & $2.15 \%$ \\
\hline High $M_{w}$ AGP & $3 \%$ & $72.7 \%$ & $55.6 \%$ & $97.4 \%$ & $11.5 \%$ & $15.4 \%$ \\
\hline Density & 1.781 & 1.702 & 1.538 & 1.641 & 1.717 & 1.703 \\
\hline $\begin{array}{l}\text { Number of } \\
\text { Regimes } \\
\text { (D-f plot) }\end{array}$ & 1 & 2 & 2 & 2 & 2 & 2 \\
\hline $\begin{array}{c}\text { Shear viscosity } \\
\eta(\mu \text { Pa.s })\end{array}$ & $699.0 \pm 27.0$ & $823.0 \pm 9.9$ & $1153.7 \pm 9.5$ & $983.8 \pm 50.7$ & $810.0 \pm 52.9$ & $806.7 \pm 29.3$ \\
\hline $\begin{array}{c}\text { Shear elastic } \\
\text { modulus } \mu(\mathrm{kPa})\end{array}$ & $41.4 \pm 2.9$ & $54.0 \pm 7.1$ & $43.2 \pm 2.2$ & $25.8 \pm 8.1$ & $32.0 \pm 4.0$ & $33.0 \pm 7.0$ \\
\hline$\Gamma_{\mathrm{QCM}-\mathrm{D}}\left(\mathrm{ng} / \mathrm{cm}^{2}\right)$ & $\begin{array}{c}277.03 \pm \\
28.33\end{array}$ & $\begin{array}{c}1375.88 \pm \\
163.20\end{array}$ & $\begin{array}{c}1051.98 \pm \\
126.57\end{array}$ & $\begin{array}{c}1609.95 \pm \\
208.5\end{array}$ & $\begin{array}{c}1232.67 \pm \\
71.73\end{array}$ & $\begin{array}{c}1264.3 \pm \\
160.0\end{array}$ \\
\hline $\mathrm{d}_{\mathrm{QCM}-\mathrm{D}}(\mathrm{nm})$ & $1.56 \pm 0.16$ & $8.08 \pm 0.96$ & $6.84 \pm 0.82$ & $9.81 \pm 1.27$ & $7.18 \pm 0.42$ & $7.42 \pm 0.94$ \\
\hline $\begin{array}{c}\text { Desorption } \\
\text { QCM-D }\end{array}$ & $7.0 \%$ & $1.2 \%$ & $5.1 \%$ & $0.3 \%$ & $2.9 \%$ & $0.9 \%$ \\
\hline$\Gamma_{\mathrm{SPR}}\left(\mathrm{ng} / \mathrm{cm}^{2}\right)$ & $34.5 \pm 1.7$ & $128.7 \pm 7.7$ & $71.7 \pm 3.6$ & $93.4 \pm 4.7$ & $86.6 \pm 4.3$ & $105.1 \pm 6.3$ \\
\hline $\mathbf{d}_{\mathrm{SPR}}(\mathrm{nm})$ & $0.19 \pm 0.02$ & $0.76 \pm 0.05$ & $0.47 \pm 0.03$ & $0.57 \pm 0.03$ & $0.50 \pm 0.02$ & $0.62 \pm 0.04$ \\
\hline Desorption SPR & $4.80 \%$ & $1.35 \%$ & $10.3 \%$ & $0.79 \%$ & $6.24 \%$ & $1.00 \%$ \\
\hline$\Gamma_{\mathrm{H} 2 \mathrm{O}}$ & $87.5 \%$ & $90.6 \%$ & $93.2 \%$ & $94.2 \%$ & $93.0 \%$ & $91.7 \%$ \\
\hline
\end{tabular}

Table S3. QCM-D and SPR calculated results, protein content $(\%$,$) High \mathrm{M}_{\mathrm{w}}$ AGP (\%,) Calculated film density and water content $\left(\Gamma_{\mathrm{H} 2 \mathrm{O}}, \%\right)$ of HIC fractions (F1, F2 and F3), IEC fractions (FA and FD) and A. senegal gum during adsorption on gold surface for a constant $\mathrm{pH} 5.0$ and ionic strength of $110 \mathrm{mM}$.

\begin{tabular}{|c|c|c|c|c|c|c|}
\hline $\begin{array}{c}110 \mathrm{mM} \\
\text { pH } 5.0\end{array}$ & HIC-F1 & HIC-F2 & HIC-F3 & IEC-F1 (FA) & IEC-F2 (FD) & A. senegal \\
\hline Protein content & $0.49 \%$ & $6.3 \%$ & $13.77 \%$ & $11.49 \%$ & $1.59 \%$ & $2.15 \%$ \\
\hline AGP Haute $M_{w}$ & $3 \%$ & $72.7 \%$ & $55.6 \%$ & $97.4 \%$ & $11.5 \%$ & $15.4 \%$ \\
\hline Density & 1.781 & 1.702 & 1.538 & 1.641 & 1.717 & 1.703 \\
\hline $\begin{array}{l}\text { Number of } \\
\text { Regimes } \\
\text { (D-f plot) }\end{array}$ & 1 & 1 & 2 & 3 & 2 & 2 \\
\hline $\begin{array}{c}\text { Shear viscosity } \\
\eta(\mu \text { Pa.s) }\end{array}$ & $1205.5 \pm 66.7$ & $1343.7 \pm 96.1$ & $1596.7 \pm 20.8$ & $1368.0 \pm 92.4$ & $1240.0 \pm 84.8$ & $\begin{array}{c}1168.0 \pm \\
113.4\end{array}$ \\
\hline $\begin{array}{c}\text { Shear elastic } \\
\text { modulus } \mu(\mathrm{kPa})\end{array}$ & $48.7 \pm 9.1$ & $64.7 \pm 8.3$ & $72.0 \pm 2.0$ & $61.5 \pm 4.8$ & $57.0 \pm 1.4$ & $47.8 \pm 7.0$ \\
\hline$\Gamma_{\text {QCM-D }}\left(\mathrm{ng} / \mathrm{cm}^{2}\right)$ & $\begin{array}{c}1770.05 \pm \\
47.42\end{array}$ & $\begin{array}{c}3446.00 \pm \\
224.67\end{array}$ & $\begin{array}{c}2610.19 \pm \\
145.91\end{array}$ & $\begin{array}{c}1864.65 \pm \\
136.53\end{array}$ & $\begin{array}{c}2725.76 \pm \\
72.66\end{array}$ & $\begin{array}{l}2213.11 \pm \\
166.46\end{array}$ \\
\hline $\mathbf{d}_{\mathrm{QCM}-\mathrm{D}}(\mathrm{nm})$ & $9.93 \pm 0.25$ & $20.23 \pm 1.32$ & $16.97 \pm 0.94$ & $11.36 \pm 0.83$ & $15.88 \pm 0.42$ & $13.01 \pm 0.98$ \\
\hline $\begin{array}{l}\text { Desorption } \\
\text { QCM-D }\end{array}$ & $10.90 \%$ & $3.48 \%$ & $3.42 \%$ & $12.0 \%$ & $2.03 \%$ & $4.75 \%$ \\
\hline$\Gamma_{\mathrm{SPR}}\left(\mathrm{ng} / \mathrm{cm}^{2}\right)$ & $445.5 \pm 22.3$ & $590.3 \pm 29.5$ & $475.5 \pm 26.3$ & $445.6 \pm 22.3$ & $509.8 \pm 21.9$ & $\begin{array}{c}361.88 \pm \\
15.90\end{array}$ \\
\hline $\mathbf{d}_{\mathrm{SPR}}(\mathrm{nm})$ & $2.50 \pm 0.13$ & $3.47 \pm 0.17$ & $3.09 \pm 0.17$ & $2.72 \pm 0.14$ & $2.97 \pm 0.13$ & $2.12 \pm 0.09$ \\
\hline Desorption SPR & $3.40 \%$ & $4.53 \%$ & $4.21 \%$ & $8.06 \%$ & $1.48 \%$ & $1.84 \%$ \\
\hline$\Gamma_{\mathrm{H} 2 \mathrm{O}}$ & $74.8 \%$ & $82.9 \%$ & $81.8 \%$ & $76.1 \%$ & $81.3 \%$ & $83.6 \%$ \\
\hline
\end{tabular}


Figure S1 - Frequency changes $(\Delta \mathrm{F})$ and Dissipation energy losses $(\Delta \mathrm{D})$ for each experimental conditions $(\mathrm{I}=$ 10 or $110 \mathrm{mM}$ ) with $(-) \mathrm{f} 3 ;(-) \mathrm{f} 5 ;(-) \mathrm{f} 7 ;(-) \mathrm{f} 9 ;(-) \mathrm{f} 11 ;(-) \mathrm{d} 3 ;(-) \mathrm{d} 5 ;(-) \mathrm{d} 7 ;(-) \mathrm{d} 9 ;(-) \mathrm{d} 11$

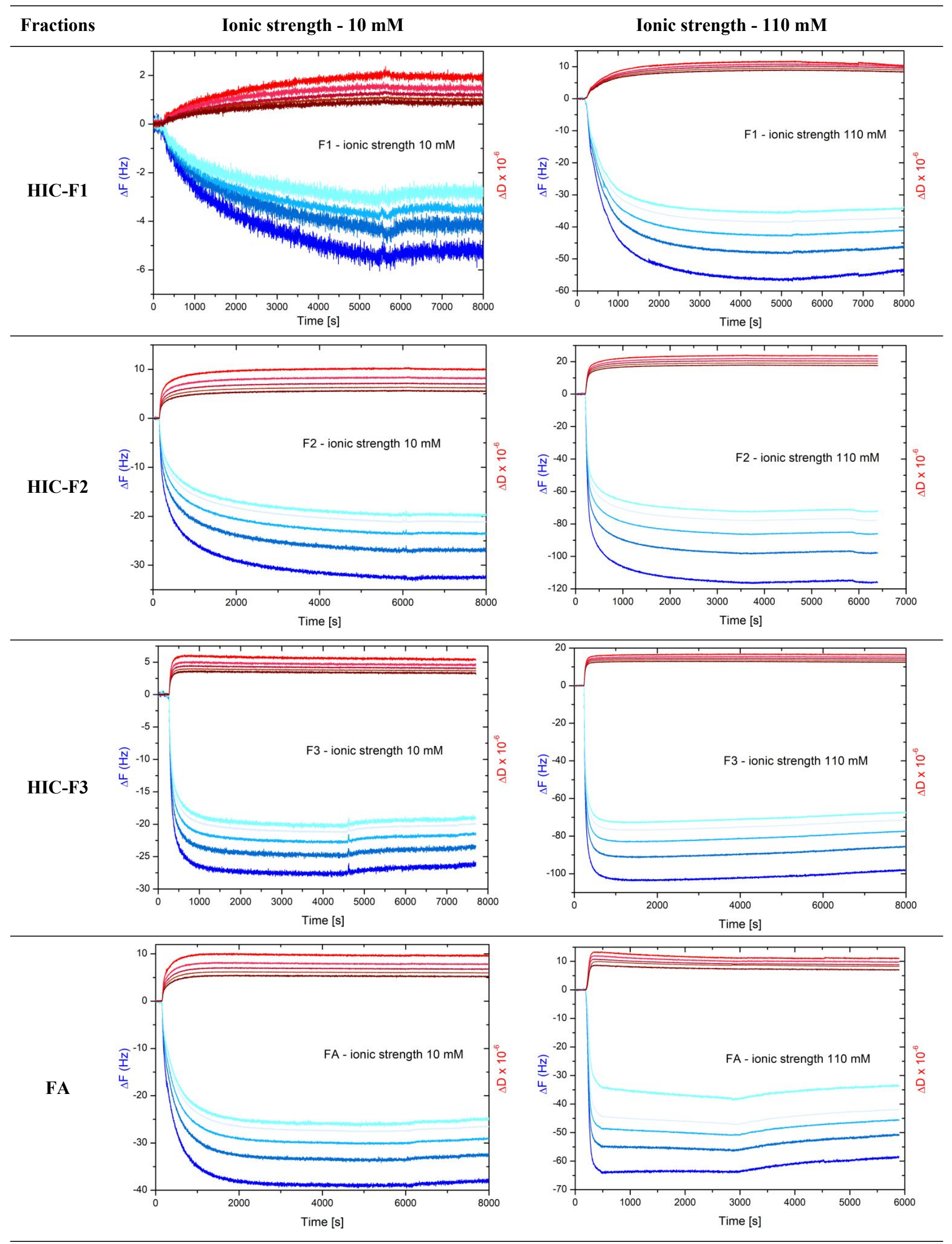



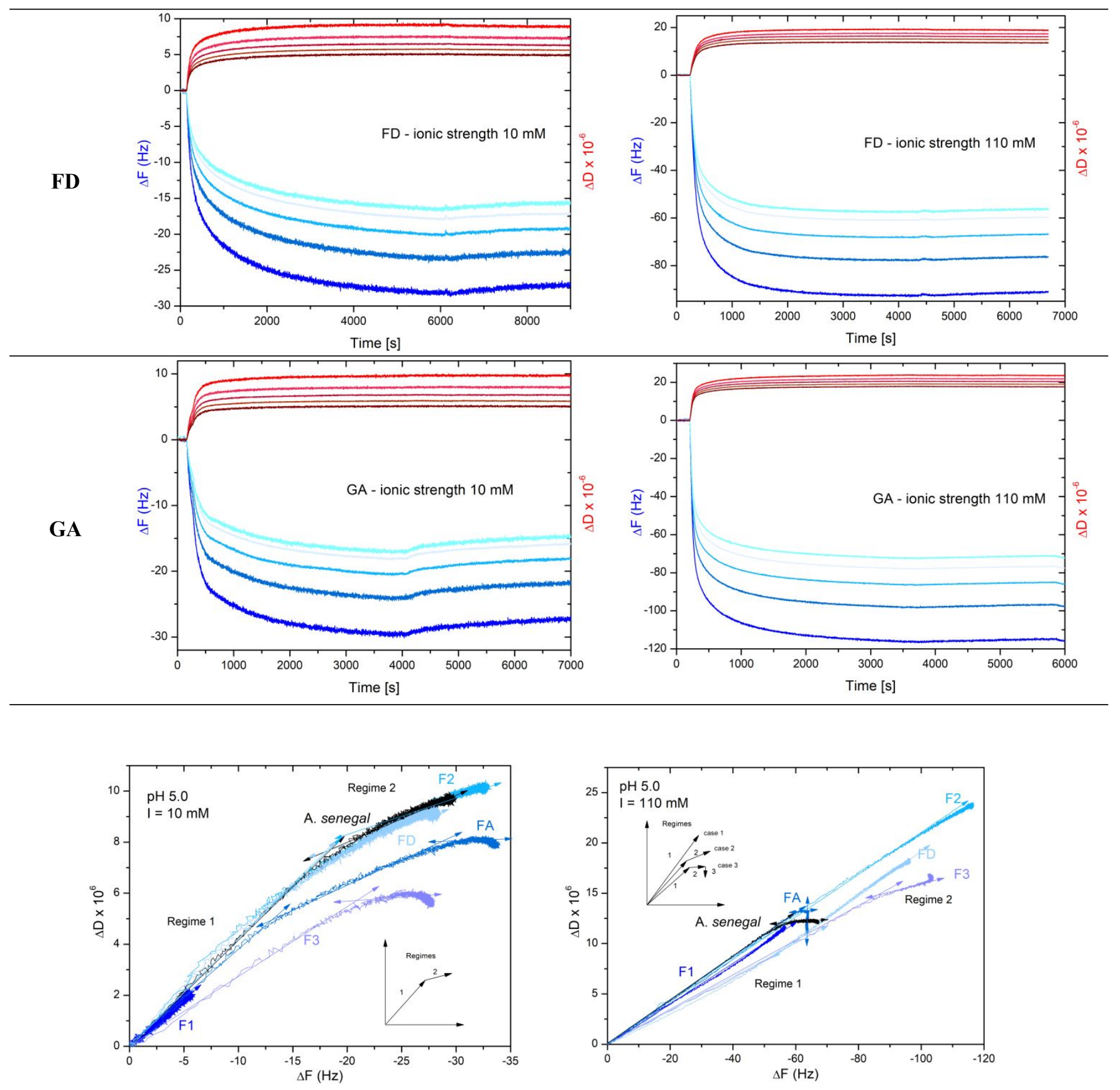

Figure S2. D-f plots of HIC and IEC fractions and details of slopes obtained with a linear fit for the two regimes at 10 and $110 \mathrm{mM}$.

Table S4. MP-SPR results

\begin{tabular}{ccccc}
\hline A. senegal & $\mathrm{d}(\mathrm{nm})$ & $\mathrm{n}_{\mathrm{a}}$ & Wavelength & Model \\
\hline $10 \mathrm{mM}$ & 0.63 & 1.48 & $670 \& 785$ & Cauchy \\
$110 \mathrm{mM}$ & $2.88 \pm 0.2$ & $1.48 \pm 0.01$ & $670 \& 785$ & Cauchy \\
\hline
\end{tabular}

\section{Experimental setup MP-SPR}

Multi-parameter surface plasmon resonance (MP-SPR) experiments were carried out for A. senegal gum on a Bionavis MP-SPR Navi ${ }^{\mathrm{TM}} 210 \mathrm{~A}$ VASA at $\mathrm{pH} 5.0$ and for two salt concentrations. The wide angular range $40-$ $78^{\circ}$ available and the use of two wavelengths $(670$ and $785 \mathrm{~nm})$ allows the direct determination of layer thickness and refractive index. The Navi ${ }^{\mathrm{TM}}$ LayerSolver software was used for the determination of refractive index of adsorbed film using both wavelength with Cauchy model. The temperature was set at $20^{\circ} \mathrm{C}$ with a flow rate of $20 \mu \mathrm{L} / \mathrm{min}$. Experiments were conducted at $10 \mathrm{mM}$ (single) and at $110 \mathrm{mM}$ (duplicate). 
Figure S3. AFM characterization of adsorbed Acacia senegal gums and its fractions layers on solid $\mathrm{SiO}_{2}$ supports at 10 and $110 \mathrm{mM}$ ionic strength. Topography, profile and phase images are presented at $5 \times 5$ (up) and $1 \times 1 \mu \mathrm{m}$ (down) for each experimental conditions in dry state.

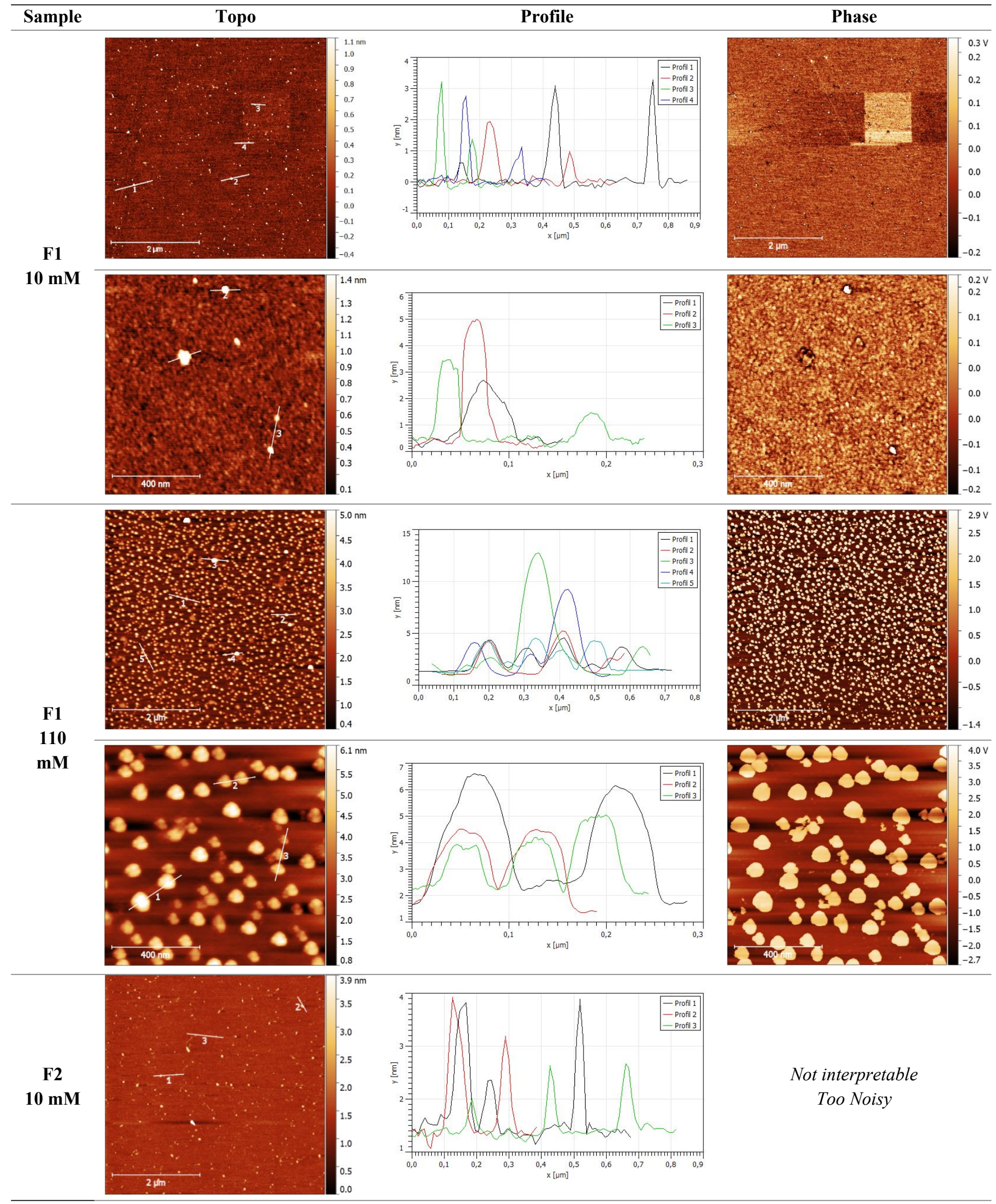




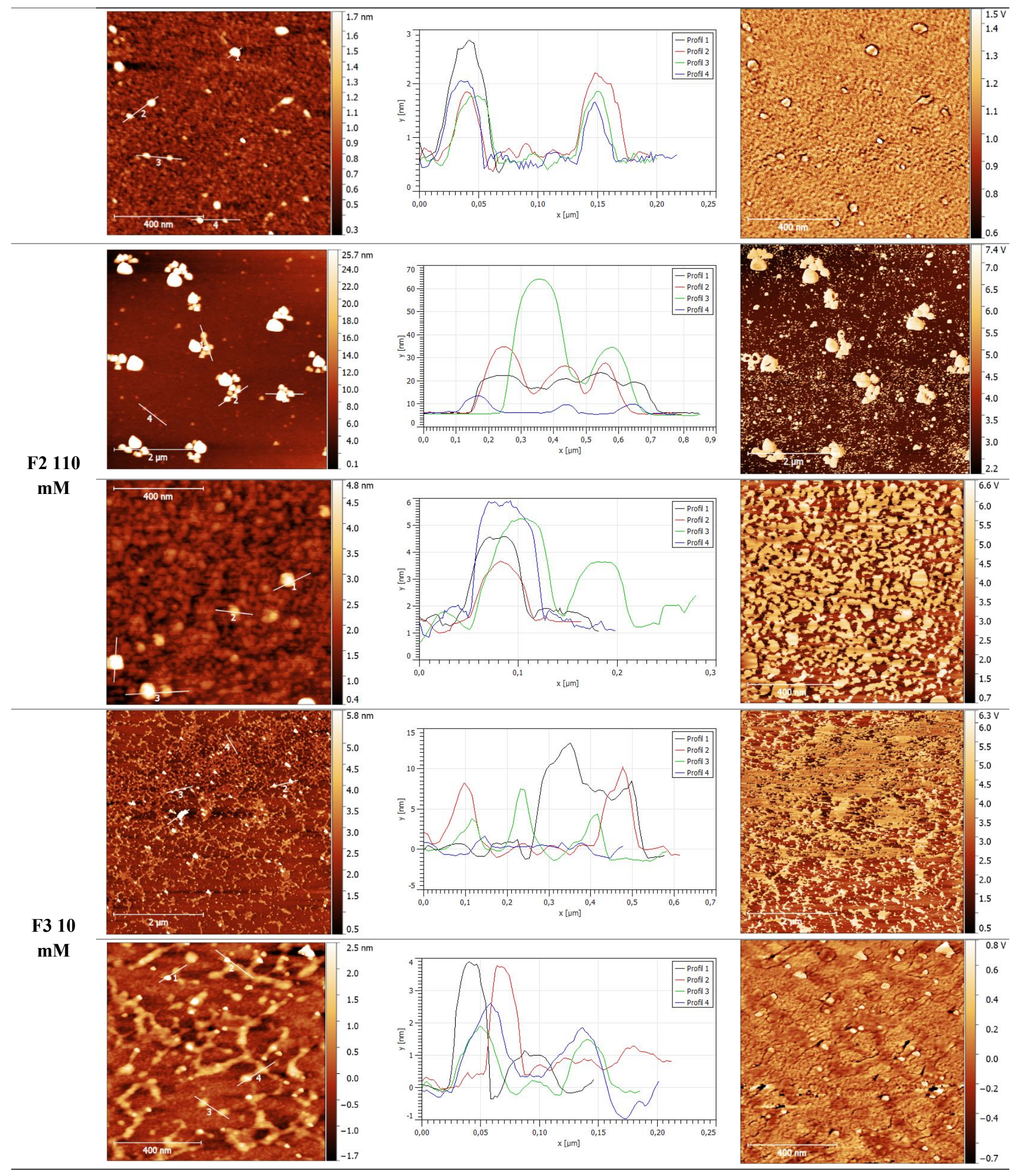




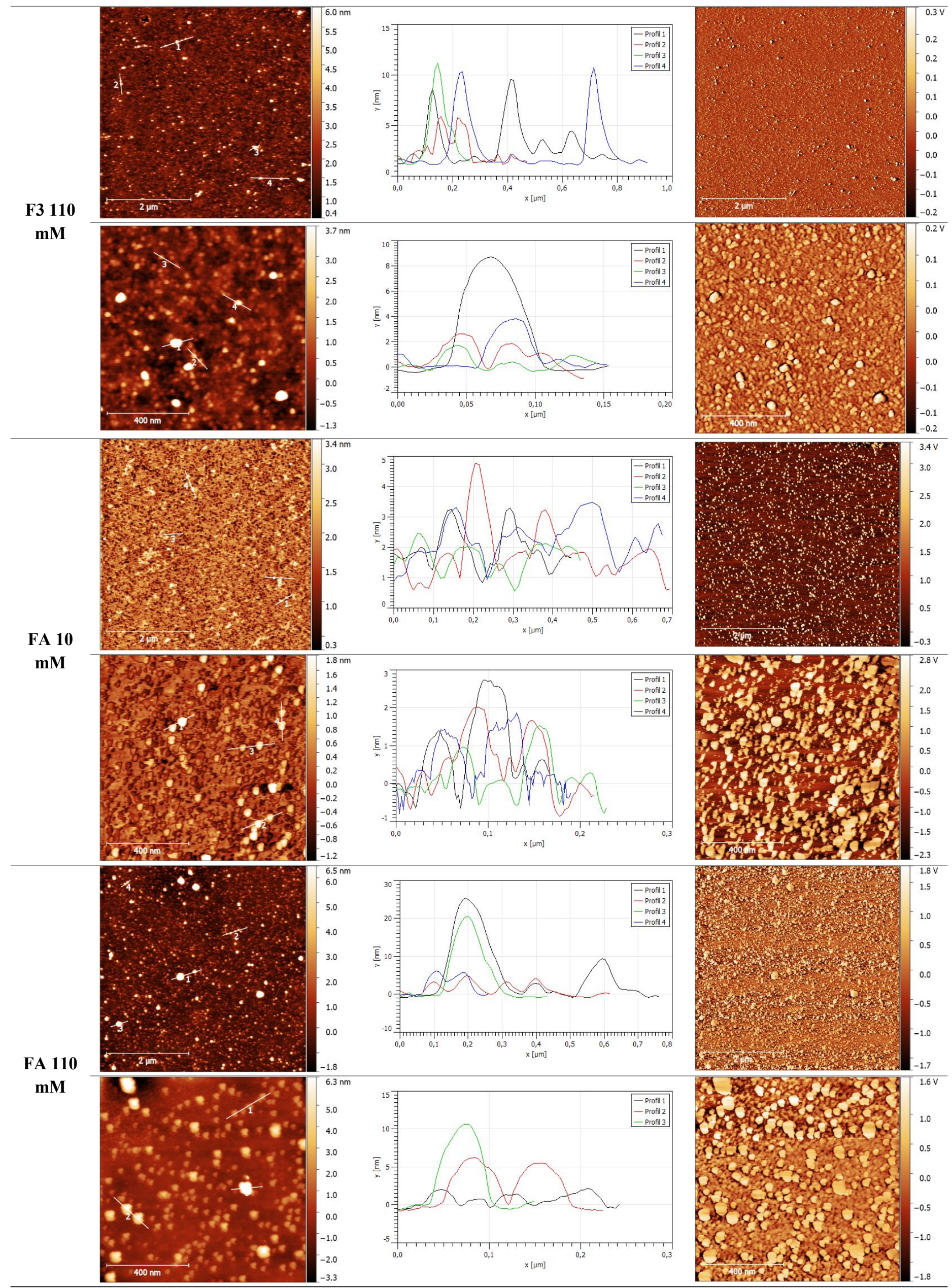




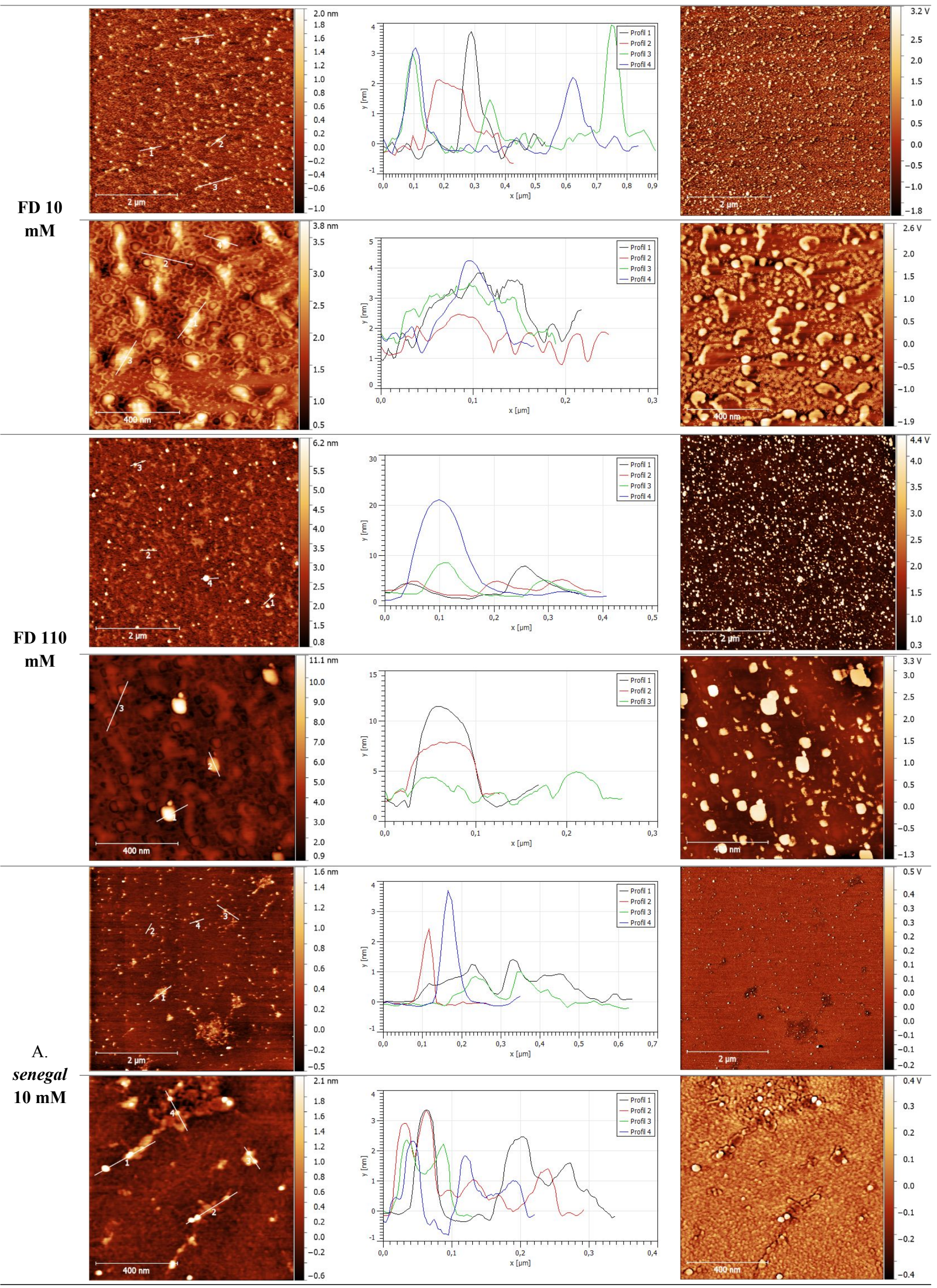




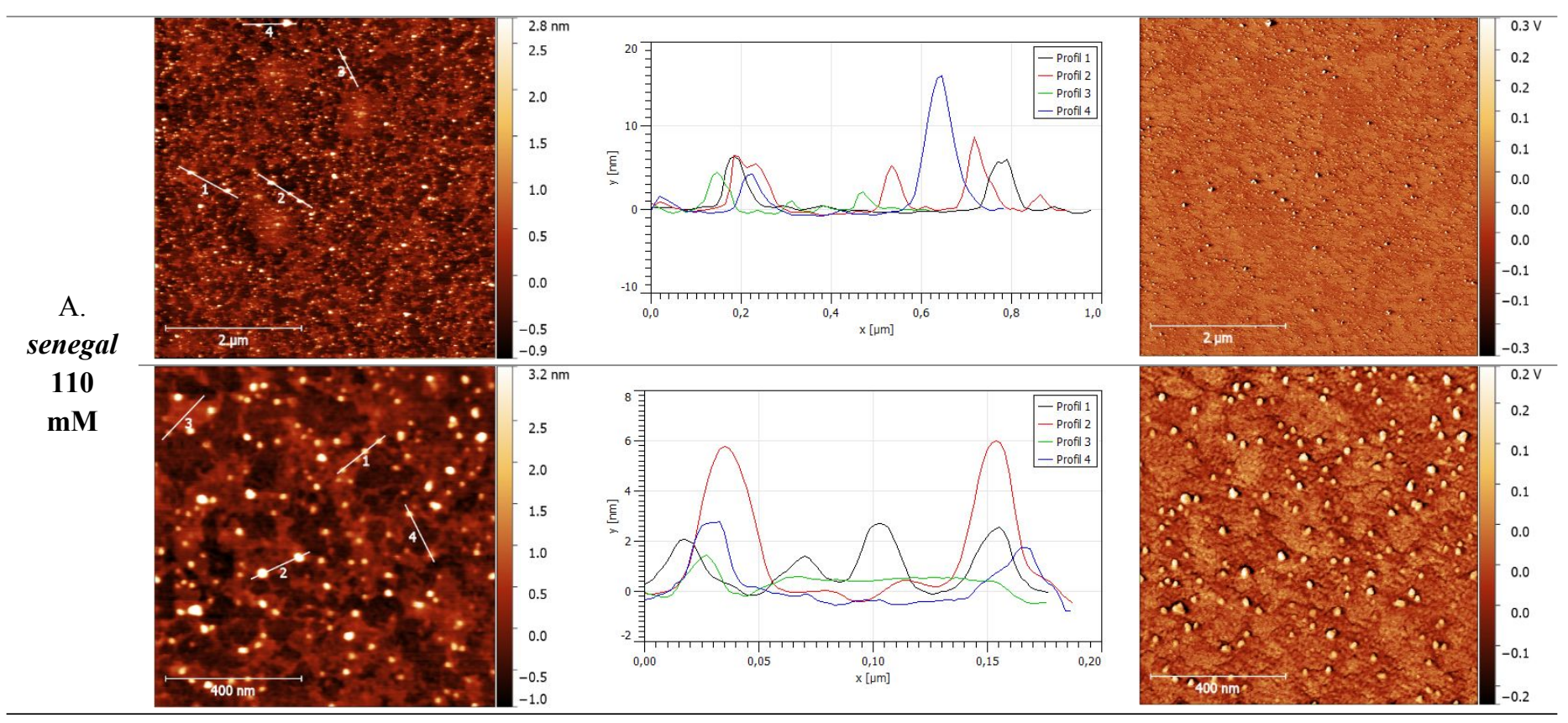

\begin{tabular}{|c|c|c|c|c|c|c|}
\hline Sample & \multicolumn{2}{|l|}{ A. senegal } & \multicolumn{2}{|l|}{ F1 } & \multicolumn{2}{|l|}{ F2 } \\
\hline $\begin{array}{c}\text { AFM } \\
\text { image } \\
5 \times 5 \mu \mathrm{m}\end{array}$ & 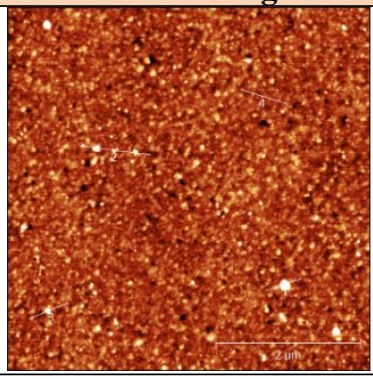 & 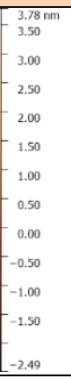 & 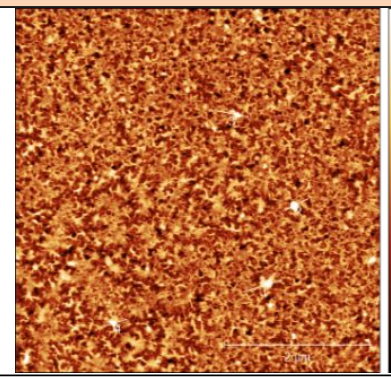 & $\begin{array}{l}6 \mathrm{~nm} \\
-5 \\
-4 \\
-3 \\
-2 \\
-1 \\
-10 \\
-1 \\
-2 \\
-2 \\
-3 \\
-4 \\
-5\end{array}$ & 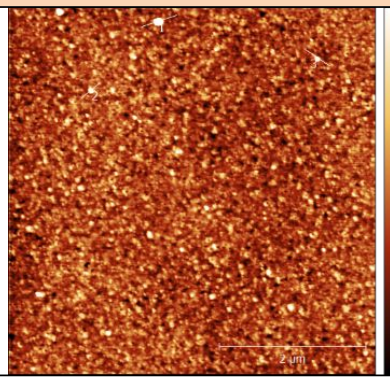 & $\begin{array}{l}5.03 \mathrm{~nm} \\
-5.00 \\
4.50 \\
-4.00 \\
-3.50 \\
-3.00 \\
-2.50 \\
-2.00 \\
-1.50 \\
0.88 \\
\end{array}$ \\
\hline $\mathbf{h}_{\mathrm{m}}(\mathbf{n m})$ & \multicolumn{2}{|l|}{3 et $5 \mathrm{~nm}$} & \multicolumn{2}{|l|}{$3.6 \mathrm{~nm}$} & \multicolumn{2}{|l|}{$2.7 \mathrm{~nm}$} \\
\hline aggregate & \multicolumn{2}{|l|}{10 to $20 \mathrm{~nm}$} & \multicolumn{2}{|l|}{10 to $20 \mathrm{~nm}$} & \multicolumn{2}{|l|}{4 to $10 \mathrm{~nm}$} \\
\hline Sample & \multicolumn{2}{|l|}{ F3 } & \multicolumn{2}{|l|}{ FA } & \multicolumn{2}{|l|}{ FD } \\
\hline $\begin{array}{c}\text { AFM } \\
\text { image } \\
5 \times 5 \mu \mathrm{m}\end{array}$ & s. & $\begin{array}{l}-3.26 \mathrm{~nm} \\
-3.00 \\
-2.50 \\
-2.00 \\
-1.50 \\
-1.00 \\
-0.50 \\
-0.00 \\
-0.50 \\
-1.00 \\
-1.50\end{array}$ & $\begin{array}{ll}8 \\
4 \\
4\end{array}$ & $\begin{array}{l}5.7 \mathrm{~nm} \\
5.0 \\
-4.0 \\
-3.0 \\
2.0 \\
-1.0 \\
-0.0 \\
-1.0 \\
-2.0 \\
-3.1 \\
\end{array}$ & 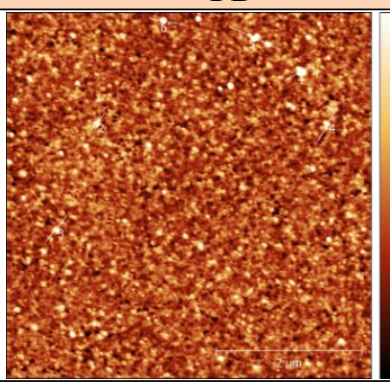 & $\begin{array}{l}3.32 \mathrm{~mm} \\
-3.00 \\
-2.50 \\
-2.00 \\
-1.50 \\
-1.00 \\
-0.50 \\
-0.00 \\
-0.50 \\
-1.00 \\
-1.50 \\
-2.47 \\
\end{array}$ \\
\hline $\mathbf{h}_{\mathrm{m}}(\mathbf{n m})$ & \multicolumn{2}{|l|}{$2.7 \mathrm{~nm}$} & \multicolumn{2}{|l|}{$3.2 \mathrm{~nm}$} & \multicolumn{2}{|l|}{$2.2 \mathrm{~nm}$} \\
\hline aggregate & \multicolumn{2}{|l|}{5 to $20 \mathrm{~nm}$} & \multicolumn{2}{|l|}{5 to $20 \mathrm{~nm}$} & \multicolumn{2}{|l|}{$>10 \mathrm{~nm}$} \\
\hline
\end{tabular}

Figure S4. AFM characterization on gold surface at pH 5.0 and $110 \mathrm{mM}$ salt concentration 
F1
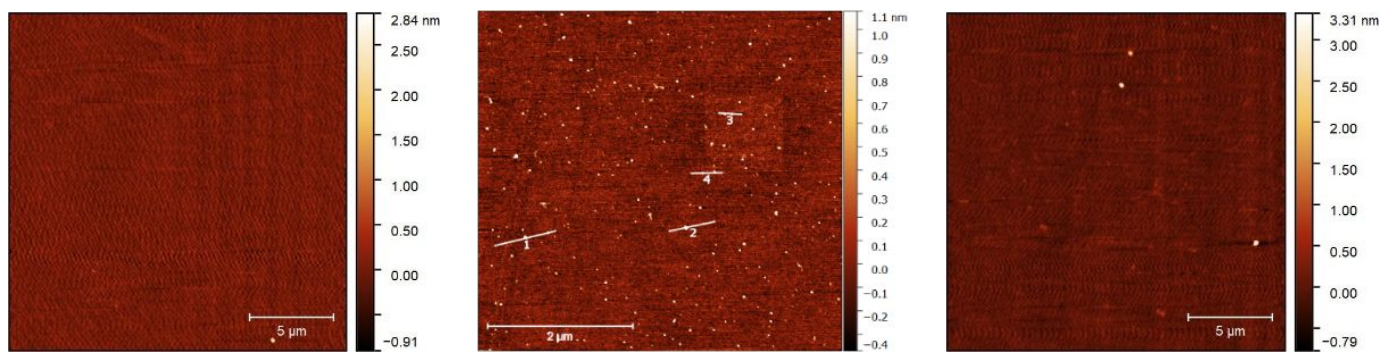

F2
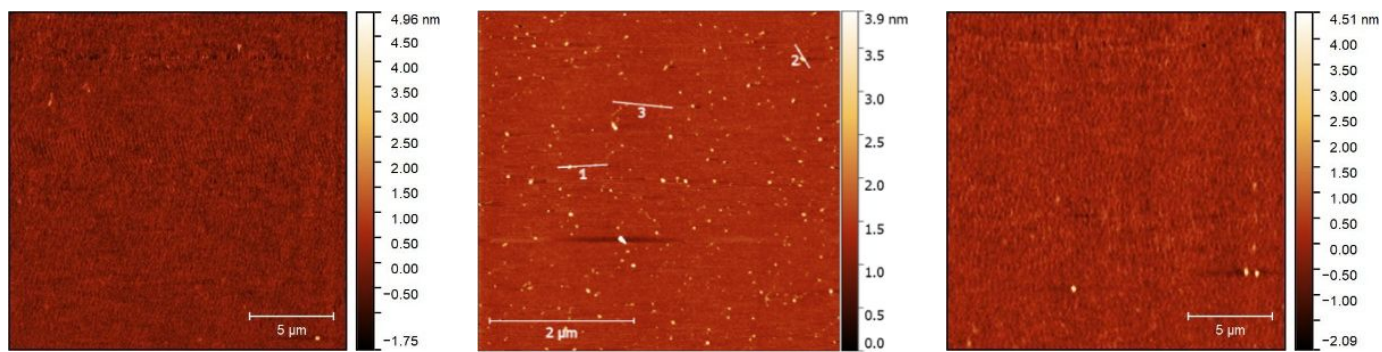

F3
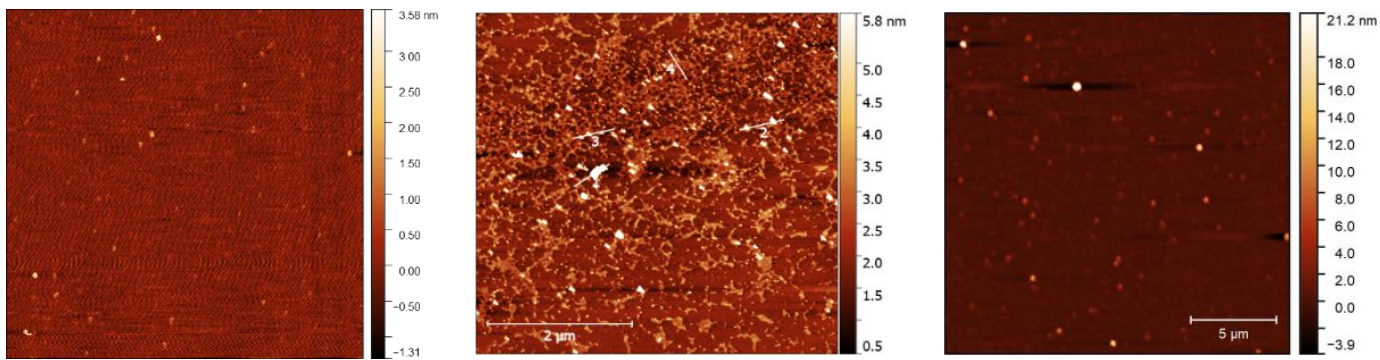

Figure S5. AFM characterization of adsorbed Acacia gums layer on solid $\mathrm{SiO}_{2}$ support, for the three $\mathrm{HIC}$ fractions F1, F2 and F3 at 500 ppm in $10 \mathrm{mM}$ acetate buffer (pH 5.0). Three states of the surface are presented: (a) after two hours of adsorption in the liquid state, (b) dry surface after adsorption and (c) surface in the liquid state after one night of rehydration

These AFM liquid images came from a series of consecutive measurements where similar observations were obtained. The noise remains continuous regardless of the analysis time or the number of measurements carried out on different locations. 

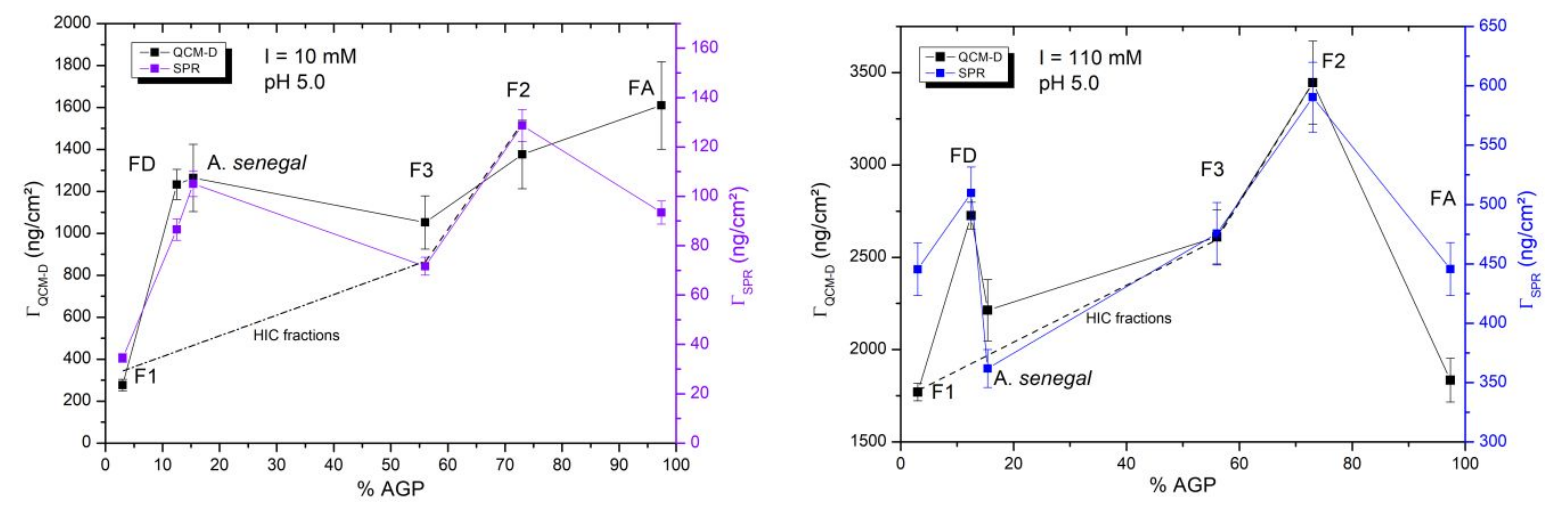

Figure S6. Adsorbed amount $\Gamma(\mathrm{ng} / \mathrm{cm} 2)$ of HIC fractions (F1, F2 and F3), IEC fractions (FA and FD) and A. senegal gum for QCM-D and SPR experiments in function of AGP high $\mathrm{M}_{\mathrm{w}}$ content at (left) $10 \mathrm{mM}$ and (right) $110 \mathrm{mM}$ salt concentration. Dashed lines connect HIC fractions.
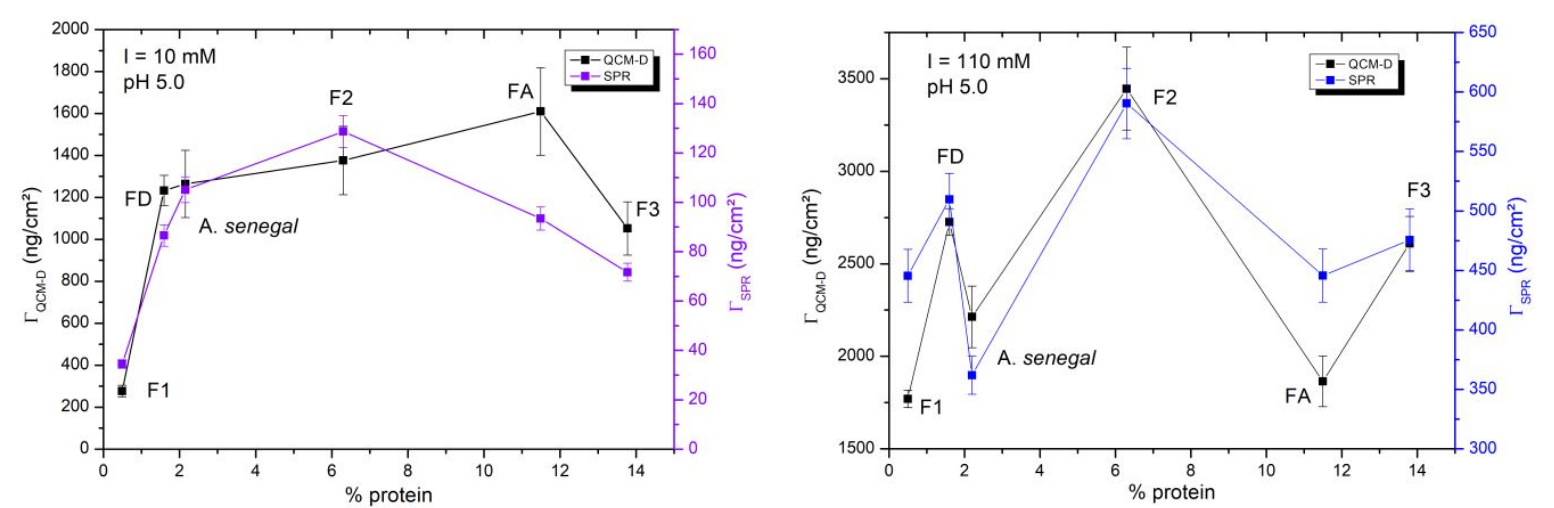

Figure S7. Adsorbed amount $\Gamma$ (ng/cm2) of HIC fractions (F1, F2 and F3), IEC fractions (FA and FD) and A. Senegal gum for QCM-D and SPR experiments in function of protein content at (left) $10 \mathrm{mM}$ and (right) 110 $\mathrm{mM}$ salt concentration. 